\title{
Prevalence and correlates of traumatic brain injury among delinquent youths
}

BRIAN E. PERRON ${ }^{1}$ AND MATTHEW O. HOWARD2, ' $U$ niversity of Michigan, School of Social Work, Ann Arbor, MI, USA; ${ }^{2}$ University of North Carolina at Chapel Hill, NC, USA

\section{ABSTRACT}

Background Delinquent youth frequently exhibit high-risk behaviours that can result in serious injury. However, little is known about traumatic brain injuries (TBIs) and their correlates in this population.

Aims To examine the period prevalence and correlates of TBIs in delinquent youths.

Method Interviews were conducted with 720 (97.3\%) residents of 27 Missouri Division of Youth Services rehabilitation facilities between March 1 and May 31, 2003. Participants [mean age $\left(\mathrm{M}_{\text {age }}\right)=15.5$, standard deviation $(\mathrm{SD})=1.2,87 \%$ male] completed measures assessing TBI, substance use, psychiatric symptoms, and antisocial traits/behaviours. TBI was defined as ever having sustained a head injury causing unconsciousness for more than 20 minutes.

Results Nearly one-in-five youths (18.3\%) reported a lifetime TBI. Youths with TBIs were significantly more likely than youths without to be male, have received a psychiatric diagnosis, report an earlier onset of criminal behaviour/substance use and more lifetime substance use problems and past-year criminal acts, evidence psychiatric symptoms, report lifetime suicidality, be impulsive, fearless, and external in locus of control and criminally victimized in the year preceding incarceration. Male gender and frequency of own criminal victimization were important predictors of TBI in multivariate analyses. Regression analyses adjusted for demographic factors, indicated that youths with TBIs were at significantly elevated risk for current depressivelanxious symptoms, antisocial behaviour, and substance abuse problems.

Conclusions TBI is common among delinquent youth and associated with wide ranging psychiatric dysfunction; however, the causal role of TBIs in the pathogenesis of co-morbid conditions remains unclear. Copyright (C) 2008 John Wiley EB Sons, Ltd. 


\section{Introduction}

Traumatic brain injuries (TBIs) range in severity from the subclinical to the fatal and are among the leading causes of morbidity and mortality in US adolescents (Hayman-Abello et al., 2003). In 2006, the National Center for Injury Prevention and Control reported that youths 15 to 19 (along with children $\leq 4$ years) were the age groups at highest risk for TBIs (Langlois et al., 2006). Falls, motor vehicle accidents, assaults, and suicide attempts (completed and non-completed) account for most TBIs in the US annually (Langlois et al., 2006).

Prior investigations suggest that delinquent youth may be at increased risk for TBI and related functional impairments. Hux et al. (1998) reported that 50\% of the delinquent youth they studied had experienced a TBI (defined as having ever received a 'blow to the head'). One-third of delinquents with TBIs were thought by their parents to have suffered adverse, long-term TBI-related effects, which diminished their ability to regulate behaviour and affect, attentional capacity, interpersonal skills, and school performance. Whereas sporting accidents were the leading cause of TBIs in non-delinquent control youth, comparatively large percentages of delinquent youth had suffered a TBI as a result of a fight, motor vehicle accident, or fall (Hux et al., 1998).

Miura et al. (2005) identified a history of TBI in only 4\% of 1336 incarcerated Japanese delinquents when a stringent criterion was employed for TBI ascertainment (i.e. head injury requiring neurological assessment and/or treatment or neurosurgical operation). Youths with TBIs showed substantial rates of electroencephalography (EEG) abnormalities and comparatively more dense family histories of drug abuse.

Craswell et al. (2004) defined TBI as a 'significant head injury involving loss of consciousness/amnesia with ongoing cognitive or social impairment' (p. 426) and reported that $27.7 \%$ of the delinquent youths they studied had TBI. Rates of lifetime TBI, depression, chronic anxiety, and substance abuse problems were significantly elevated in delinquent youth compared to non-delinquents.

With regard to the reported prevalence of TBI in delinquent samples, Hux et al. (1998) noted that different definitions of TBI and significant variations in the severity and presentation of TBI (particularly the finding that an estimated 75\% of TBIs are mild and may not ever come to clinical attention) (Caveness, 1979; Miller and Jones, 1985; Kraus and Nourjah, 1988), render precise estimates diffcult to establish. To these reasons they added the reluctance of many TBI victims and their family and peer associates to report injuries due to abuse, violence, and intoxication and otherwise noted that many youth are not aware that 'minor' blows to the head can result in brain damage, particularly if they are recurrent.

Although few longitudinal studies have examined the causes or consequences of TBI in delinquent youth, Rantakallio et al. (1992) found a significantly elevated incidence of delinquency among youth who had experienced brain trauma before age 14 in a large study of Finnish youth. Other investigations have identified 
persistent memory deficits, neuropsychiatric impairments, and psychosocial problems in persons experiencing even mild TBIs (Butler et al., 1981; Rimel et al., 1981; Asarnow et al., 1991; McAllister, 1992; Rivara et al., 1994; Bloom et al., 2001), although the causal ordering of such associations remains uncertain.

In light of the exigent need for additional research examining associations between TBI and adolescent antisocial behaviour, we: (1) examined the prevalence of TBI in a state population of incarcerated adolescents, (2) identified factors associated with elevated risk for TBI, and (3) evaluated whether and to what extent a history of TBI is associated with substance use problems, psychiatric symptoms, and antisocial behaviour.

\section{Methods}

\section{Survey procedures}

We surveyed all young people resident in the Missouri Division of Youth Services (DYS) between March 1 and May 31, 2003. The Missouri DYS is the legal guardian of all residents who are committed to its care by the state's 45 juvenile courts. Ten adolescents were on furlough at the time of interviewing and two adolescents were transferred to another facility while interviewers were at the facility, but before they could be interviewed. Of the 728 adolescents available for interview, all agreed to participate. However, five interviews were discontinued; four adolescents displayed signs or reported symptoms of psychosis and one adolescent chose not to continue. The 723 adolescents who completed the interview constituted $97.7 \%$ of DYS residents at the time interviewing was conducted, $99.3 \%$ of residents available for interviewing, and approximately $55.0 \%$ of adolescents committed to DYS care in the prior year. Thus, the present study is virtually a census of the population of DYS residents at the time the study was undertaken and a large, representative sample of DYS annual residents.

Participation in the study was voluntary. Face-to-face interviews of all adolescents were conducted using a comprehensive solvent assessment inventory. All interviewers completed an intensive one-day training session and an interview editor was on-site at each facility as adolescents were interviewed to minimize interviewer omissions and errors. DYS residents are under 24-hour-a-day supervision; thus, interviews were conducted in large rooms that provided private areas where confidential interviews could be conducted simultaneously with between three and six adolescents.

The sample recruitment protocol ensured that no adolescents who had completed the interview at one facility repeated completion at another facility. This study was approved by DYS, the Washington University Human Studies Committee Institutional Review Board, the Federal Office of Human Research Protection, and was granted a Certificate of Confidentiality by the National 
Institute on Drug Abuse (NIDA). Adolescents received $\$ 10.00$ for their participation.

\section{Measures}

\section{Traumatic brain injury (TBI)}

TBI was identified using the following self-report survey item: 'In your lifetime, have you ever had a head injury that caused you to black out for more than 20 minutes?' (yes/no).

\section{Substance use problems}

Lifetime substance-related problems were assessed with the eight-item Alcohol/ Drug Use Scale of the Massachusetts Youth Screening Instrument - Second Version (MAYSI-2) developed for use with juvenile justice populations (Grisso and Barnum, 2000). Scores could range from zero to eight (Grisso and Barnum, 2000). Grisso and Barnum (2000) found the scale to be internally consistent $(\alpha=0.86)$ in their norming sample; the $\alpha$ coefficient in this study was 0.83 . Youths were also asked about lifetime use (yes/no) of heroin, cocaine or crack cocaine, marijuana, and ecstasy.

\section{Psychiatric variables}

Youths were asked whether they had ever been diagnosed with a mental illness by a psychiatrist or other doctor (yes/no). Youths also completed the Brief Symptom Inventory (BSI) (Derogatis, 1993). Five BSI subscales were included in this study: depression, anxiety, phobic anxiety, interpersonal sensitivity, and psychoticism. The $\alpha$ coefficients for these subscales ranged from 0.70 to 0.83 .

Adolescents completed the five-item MAYSI-2 suicide ideation scale, which requires adolescents to respond 'yes' or 'no' to questions assessing whether or not they have ever wished they were dead, have felt like life was not worth living, have felt like hurting themselves, have felt like killing themselves, and have ever given up hope for their life (Grisso and Barnum, 2000). Grisso and Barnum, (2000) reported a $\alpha$ coefficient of 0.83 ; the $\alpha$ coefficient in this study was 0.91 .

Study participants also completed the 56-item Psychopathic Personality Inventory Short-Version (PPI-SV) (Lilienfeld and Andrews, 1996). Four subscales from the PPI-SV were included in this study: fearlessness, impulsive non-conformity, coldheartedness, and blame externalization. The PPI-SV has been used to assess antisocial traits in a variety of offender populations and has shown acceptable psychometric properties (Edens et al., 2001).

\section{Delinquent behaviour}

The Self-Report of Delinquency (SRD) (Elliot et al., 1989) was used to assess how many times in the year before they were incarcerated youth engaged in seven 
non-violent and 10 violent crimes. Responses could range from zero (never) to eight (2-3 times a day) for each item. Total SRD scale scores could range from 0 to $136(\alpha=0.84)$, while the ranges of possible scores were $0-56(\alpha=0.81)$ and 0-80 ( $\alpha=0.73)$ for the non-violent and violent offense subscales, respectively. Youths also reported the age at which they first committed a criminal offence.

\section{Victimization}

A four-item Victimization Index was used to assess personal experiences of criminal victimization in the year prior to incarceration. The response format for the victimization scale was identical to that used for the SRD. However, due to high skewness, these items were dichotomized to indicate the presence or absence of each specific type of experience. These specific items included: 'been hit by someone trying to hurt you,' 'had someone use a weapon or force to get money or things,' 'been attacked by someone with a weapon or by someone trying to seriously hurt or kill you,' and 'had some of your things stolen from you.' In the bivariate analyses, these items were examined individually. In the multivariate analysis, they were used as a four-item index, with scores ranging from zero to four.

Adolescents also completed four items from the MAYSI-2 Traumatic Experiences scale. These items assessed whether or not a youth had ever seen someone severely injured or killed (in person, not in the movies or on television), had a lot of bad thoughts or dreams about a bad or scary event that happened to them, had ever been badly hurt or in danger of getting badly hurt or killed, and had ever in their whole life had something very bad or terrifying happen to them. Each item was dichotomously scored, resulting in a scale range of zero to four. A higher score indicates a higher level of exposure to traumatic experiences. This measure exhibits acceptable psychometric properties (Grisso and Barnum, 2000). Reliability in this study was 0.69.

Demographic and psychosocial variables

Gender, age, self-reported racial status, family receipt of public assistance, and geographical area of family residence (i.e. urban, suburban, small town, rural) were recorded for each youth. Youths were also asked to estimate the age at which they committed their first crime.

\section{Analytic procedures}

Less than $1 \%$ of the total data points were missing. Three participants did not respond to the survey question measuring TBI. These participants were excluded from the analysis, which resulted in a sample size of 720 . The remaining missing values were imputed with the aregImpute function in the HMisc package for $\mathrm{R}$ (Harrell, 2002). 
Chi-square tests were used to test the association between TBI and the categorical variables, and effect sizes were summarized with phi coefficients $(\Phi)$. A multivariate analysis of variance was used to test the association between TBI and continuous variables. Effect sizes were summarized using point biserial correlations $\left(r_{\mathrm{pb}}\right)$ based on their respective bivariate relationships.

Multivariate logistic regression was used to identify factors associated with TBI risk. TBI was also assessed as an independent variable in order to examine the associations of TBI with various functional or behavioural impairments. Multivariate negative binomial regression was used for highly skewed outcomes (i.e. BSI - depressive symptoms, BSI - anxiety symptoms, SRD delinquency), and multivariate linear regression was used to examine lifetime substance use problems, which was normally distributed. These variables were selected based on existing literature and significant bivariate associations observed in this study.

\section{Results}

\section{Sample description}

Approximately 55\% $(n=399)$ of the sample was White and $45 \%(n=321)$ was non-White. Most (87\%) of the sample was male, and ages ranged from 11 to 20 $[$ mean $=15.5$, standard deviation $(S D)=1.24$, median $=16]$. Forty-one percent $(n=292)$ of respondents reported that their family received public assistance. Prior to being incarcerated, $53 \%(n=382)$ of youth lived in urban/suburban areas. The age at commission of first crime ranged from 4 to 16 (mean $=10.6, \mathrm{SD}=$ 2.8 , median $=11)$. Approximately $52 \%(n=371)$ reported having received a formal diagnosis of mental illness.

\section{Prevalence and correlates of TBI}

Bivariate associations

More than $18 \%(n=132)$ of the study sample reported a TBI. Occurrence of TBI was significantly associated with a history of a mental illness $\left(\chi^{2}[1]=10.94, p<\right.$ 0.001); however, the effect size for this relationship was small $(\Phi=0.12)$. Specifically, 64\% of respondents with TBI had a history of diagnosis with mental illness compared to $49 \%$ of those without. TBI status was not associated with ethnicity, urbanicity of familial residence or receipt of welfare. Males were disproportionately represented among youths who reported histories of TBI $(\mathrm{TBI}+=93 \%$ male; TBI- $=85 \%$ male $)\left(\chi^{2}[1]=5.54, p=0.019\right)$, although the effect size for gender was small $(\Phi=0.09)$.

Respondents with TBI were significantly more likely than their counterparts without it to have used heroin (11\% versus 5\%), cocaine or crack cocaine (36\% versus $21 \%$ ), marijuana (93\% versus $85 \%$ ), and ecstasy ( $33 \%$ versus $17 \%$ ). 
Respondents with TBI were also significantly more likely than those without to report being hit by someone (89\% versus $74 \%$ ), have someone use a weapon or force to get money or other things from them (51\% versus 32\%), and be attacked by someone with a weapon or someone who was otherwise trying to injure them (72\% versus $45 \%$ ) (Table 1 ).

Associations between TBI and continuous variables were tested using MANOVA. The results of this analysis and the bivariate associations are summarized in Table 2 . Wilk's lambda $(\lambda)$ was used to examine the main effect. The Wilk's lambda value was significant, indicating global differences between groups. All variables included in this test were significantly associated with TBI except age and the PPI coldheartedness subscale. The effect sizes $\left(r_{\mathrm{pb}}\right)$ ranged from $10.04 \mid$ to $|0.27|$. The variables with the strongest relationships to TBI were the psychiatric, substance use problems, and delinquency measures.

\section{Exploratory mutlivariate analysis}

TBI risk factors

An exploratory, three-step multivariate logistic regression model was specified to identify risk factors for TBI. Demographic variables (i.e. age, ethnicity, family residence, welfare, and gender) were entered in the first step, yielding a marginally significant likelihood ratio (LR) chi-square test ( $\left.\operatorname{LR} \chi^{2}[5]=7.7, p=0.05\right)$. In the second step, additional youth characteristics were entered into the model, including the MAYSI-2 substance use problems and suicidality scales; SRD Delinquency Index; age at commission of first crime; age at initiation of alcohol or marijuana use; BSI anxiety and depression subscales; and PPI impulsive non-conformity and fearlessness subscales. This subset of variables was selected on the basis of their bivariate associations with TBI, efforts to avoid multicollinearity, and clinical importance of the assessed attributes. The overall model was significant (LR $\chi^{2}$ $[14]=75.35, p<0.001$, pseudo $\left.R^{2}=0.16\right)$. In this model, three variables were significantly associated with TBI: gender [male, odds ratio $(\mathrm{OR})=2.61,95 \%$ confidence interval $(\mathrm{CI})=1.19-5.73]$, frequency of delinquent behaviours in prior year $(\mathrm{OR}=1.17,95 \% \mathrm{CI}=1.03-1.33)$, and BSI anxiety (log transformed, $\mathrm{OR}=2.25$, 95\% CI $=1.12-4.49$ ).

In the third step, the victimization and MAYSI-2 traumatic experiences indices were entered into the model. The overall model was significant (LR $\chi^{2}$ [16] $=99.17, p<0.001$, pseudo $\left.R^{2}=0.21\right)$, but only two variables were associated with TBI risk: gender (male, $\mathrm{OR}=2.59,95 \% \mathrm{CI}=1.17-5.74$ ) and the MAYSI-2 traumatic experiences index $(\mathrm{OR}=1.57,95 \% \mathrm{CI}=1.28-1.23)$. It should be noted that the victimization and traumatic experiences index measures were included in the third step because of their possible proximal association with TBI. This provided the opportunity to assess how the other variables were associated with TBI risk in absence of these important factors. 
Table 1: Chi-square analyses testing associations between TBI and demographic, psychosocial, substance use, and victimization variables

\begin{tabular}{|c|c|c|c|c|c|}
\hline Variable & $\begin{array}{l}\text { Head injury - no } \\
(N=588) N(\%)\end{array}$ & $\begin{array}{l}\text { Head injury - yes } \\
(N=132) N(\%)\end{array}$ & $\chi^{2}(\mathrm{df})$ & $p$ & $\Phi$ \\
\hline \multicolumn{6}{|l|}{ Gender } \\
\hline Male & $503(85.5)$ & $123(93.2)$ & $5.54(1)$ & 0.019 & 0.09 \\
\hline Female & $85(14.5)$ & $9(6.8)$ & & & \\
\hline \multicolumn{6}{|l|}{ Ethnicity } \\
\hline White & $328(55.8)$ & $71(53.0)$ & $0.33(1)$ & 0.566 & 0.02 \\
\hline Non-white & $260(44.2)$ & $61(47.0)$ & & & \\
\hline \multicolumn{6}{|l|}{ Urbanicity } \\
\hline Urban / suburban & $310(52.7)$ & $72(54.5)$ & $0.14(1)$ & 0.704 & 0.01 \\
\hline Rural / small town & $278(47.3)$ & $60(45.5)$ & & & \\
\hline \multicolumn{6}{|l|}{ Welfare } \\
\hline Yes & $242(41.2)$ & $50(38.0)$ & $0.48(1)$ & 0.488 & 0.03 \\
\hline No & $346(58.4)$ & $82(62.1)$ & & & \\
\hline \multicolumn{6}{|c|}{ History of a mental illness } \\
\hline Yes & $285(48.7)$ & $85(64.4)$ & $10.94(1)$ & $<0.001$ & 0.12 \\
\hline No & $303(51.5)$ & $47(35.6)$ & & & \\
\hline \multicolumn{6}{|l|}{ Lifetime heroin use } \\
\hline Yes & $32(5.4)$ & $15(11.4)$ & $6.19(1)$ & 0.013 & 0.09 \\
\hline No & $556(94.6)$ & 117 (88.6) & & & \\
\hline \multicolumn{6}{|c|}{ Lifetime crack or cocaine use } \\
\hline Yes & $121(20.6)$ & $48(36.4)$ & & & \\
\hline No & $467(79.4)$ & $84(63.6)$ & $14.95(1)$ & $<0.001$ & 0.14 \\
\hline \multicolumn{6}{|c|}{ Lifetime marijuana use } \\
\hline Yes & $501(85.2)$ & $123(93.2)$ & $5.27(1)$ & 0.015 & 0.09 \\
\hline No & $87(14.8)$ & $9(6.8)$ & & & \\
\hline \multicolumn{6}{|l|}{ Lifetime ecstasy use } \\
\hline Yes & $99(16.8)$ & $43(32.6)$ & $16.87(1)$ & $<0.001$ & 0.15 \\
\hline No & $489(83.2)$ & $89(67.4)$ & & & \\
\hline \multicolumn{6}{|c|}{ Been hit by someone trying to hurt you } \\
\hline Yes & $437(74.3)$ & $118(89.4)$ & $13.87(1)$ & $<0.001$ & 0.14 \\
\hline No & $151(25.7)$ & $14(10.6)$ & & & \\
\hline \multicolumn{6}{|c|}{ Had someone use a weapon or force to get money or things } \\
\hline Yes & $187(31.8)$ & $67(50.8)$ & $16.96(1)$ & $<0.001$ & 0.15 \\
\hline No & $401(68.2)$ & $65(49.2)$ & & & \\
\hline
\end{tabular}

Been attacked by someone with a weapon or by someone trying to seriously hurt or kill you

$\begin{array}{lcrrrr}\text { Yes } & 267(45.4) & 95(72.0) & 30.42(1) & <0.001 & 0.21 \\ \text { No } & 321(54.6) & 37(28.0) & & & \\ \text { Had some of your things stolen from you } & & & & & \\ \text { Yes } & 467(79.4) & 115(87.1) & 4.45(1) & <0.035 & 0.08 \\ \text { No } & 121(20.6) & 17(12.9) & & & \end{array}$

Note: Statistically significant values $(p<0.05)$ are represented in italic typeface. All percentages are column percentages. 


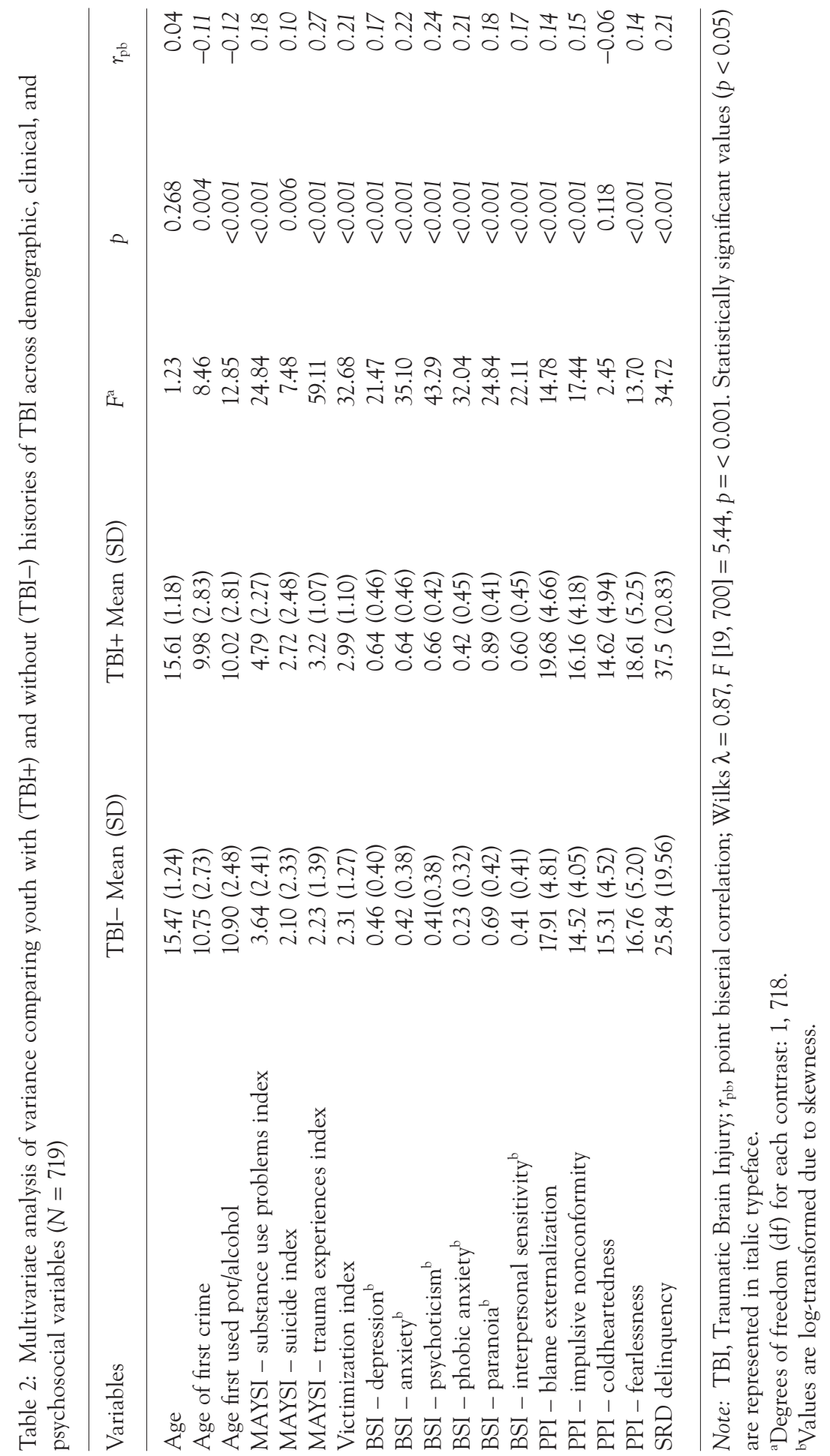




\section{Psychosocial and clinical outcomes}

Four exploratory, two-step regression models were specified to examine the relationships between TBI and psychosocial/clinical outcomes (i.e. current depressive symptoms, current anxiety symptoms, past-year total delinquency, and lifetime substance use problems), while controlling for potentially confounding factors (e.g. age, gender, ethnicity, and urbanicity). In the first step, outcomes were examined by including demographic factors and TBI in the models. Each model exhibited a good fit with the data, and the association between TBI and each outcome was significant. However, after controlling for other study variables (see Table 1), the significant associations between TBI and each outcome assessed were non-significant.

\section{Discussion}

We found a high rate of TBI in this delinquent group, nearly one in five of whom reported a potentially clinically important brain injury. Prior research on TBI has revealed an incidence ranging from about 180-250 per 100,000 in the US in population-based studies (Bruns and Hauser, 2003). Research also shows comparatively high levels of TBI for adolescents and young adults - that is, up to 415 per 100,000 (Bruns and Hauser, 2003). Our findings, however, approximate to those in the work by Craswell et al. (2004), which may be attributable to the similarity of the two studies vis-à-vis measurement of TBI (i.e. significant head injury with attendant loss of consciousness). Given the young average age of respondents in our study, it is likely that the lifetime prevalence of TBI will continue to climb as these youth (virtually the entire population of all incarcerated Missouri youth at the time the study was conducted) enter early and middle adulthood.

Although the reported cross-sectional findings do not allow for strong aetiological inferences to be drawn vis-à-vis causes and consequences of TBI, it is clear that TBI often occurs as one among a constellation of adversities affecting such youths. At the bivariate level, youths with TBI did not differ significantly from those without with respect to demographic factors other than gender. Like Hux et al. (1998), we found boys at higher risk for TBI than girls. Youths with TBI, however, displayed significantly more current psychiatric distress on virtually all subscales of the BSI, significantly earlier onset of criminal and substance-using behaviours, more lifetime substance abuse problems and suicidality, and more frequent past-year criminality than youths without TBI. Whether these problems and/or the impulsive and fearless temperaments of youths with TBI antedated and possibly contributed to TBI or were a result of TBI, or some combination thereof, is unclear.

Bivariate analyses indicated that measures of past year violent victimization and frequency of antisocial behavior, as well as lifetime exposure to violent trau- 
matic events were among the strongest correlates of TBI in this population of delinquent youth. These findings suggest that fights and other assaults may have been a significant source of TBIs in this population. Bivariate and multivariate analyses revealed that gender and extent of lifetime exposure to traumatic events were the strongest predictors of TBI status.

In analyses adjusting for demographic factors, TBI+ status significantly predicted higher levels of lifetime substance abuse problems, current distress due to depressive and anxious symptoms, and frequency of criminal behaviour in the year preceding incarceration. The effect sizes associated with these findings were modest to moderate in magnitude. When co-linear covariates were entered into the equations, results for TBI became non-significant. Prior research has shown that some mild TBI may not lead to neuropsychiatric impairment. For example, a prospective multisite study revealed that functional outcomes after isolated mild TBI are generally good-to-excellent for both elderly and younger patients, with younger patients requiring less time to recover and inpatient treatment time (Mosenthal et al., 2004; see also Belanger et al., 2005). This study did not assess the severity of TBI or treatment received following it. Thus, some youth with more severe TBI and unmet treatment need may have greater functional impairments than the overall trends suggest. The causal structure of associations between TBI and diverse neuropsychiatric co-morbidities remains unclear and their elucidation must await long-term longitudinal investigation of these relationships. This is particularly relevant as prior research has suggested that mild head injuries may be the consequence rather than the cause of psychological deviancy. At present, it is important for clinicians who work with delinquent youth to make systematic inquiries about head injury and have a high index of suspicion for characteristic co-morbid conditions when youths report such histories.

Limitations of this study included the reliance on self-report for determination of lifetime TBI history, absence of detailed information such as medical records regarding the severity of TBI, information about mode of TBI, and timing of TBI relative to onset of associated problems and behaviours. Ideally, self-reports in this study would have been triangulated with additional information, which was beyond the scope of the original study. Although self-reports may be subject to bias, it has been long recognized that they are among the most useful measures of criminal behaviours and a key method of meeting the needs of descriptive and etiological research (see Huizinga and Elliot, 1986). Other researchers have found the validity of self-reports of offending to be high, especially for drug offences and males (Jolliffe et al., 2003; see also Maxfield et al., 2000; Krischer et al., 2007). Despite the limitations of this study, we believe the large sample (virtually a population census), high participation rate, and comprehensive assessment approach are strengths of this study and that the findings will be useful to practitioners working with delinquent youths. 


\section{References}

Asarnow R, Satz P, Light R, Lewis R, Neumann E (1991) Behavior problems and adaptive functioning in children with mild and severe closed head injury. Journal of Pediatric Psychology 16: $543-555$.

Belanger HG, Curtiss G, Demery JA, Lebowitz BK, Vanderploeg RD (2005) Factors moderating neuropsychological outcomes following mild traumatic brain injury: a meta-analysis. Journal of the International Neuropsychology Society 11: 215-227.

Bloom D, Levin H, Ewing-Cobbs L, Saunders A, Song J, Fletcher JM, Kowatch R (2001) Lifetime and novel psychiatric disorders after pediatric traumatic brain injury. Journal of the American Academy of Child and Adolescent Psychiatry 40: 572-579.

Bruns J, Hauser WA (2003) The epidemiology of traumatic brain injury: a review. Epilepsia 44: 2010.

Butler G, Chadwick O, Shaffer D, Rutter M, Traub M (1981) A typology of psychosocial functioning in pediatric closed-head injury. Child Neuropsychology 3: 98-133.

Caveness W (1979) Incidence of craniocerebral trauma in the United States in 1976 with trend from 1970-1975. Advances in Neurology 22: 1-3.

Craswell K, Maughan B, Davis H, Davenport F, Goddard N (2004) The psychosocial needs of young offenders and adolescents from an inner city area. Journal of Adolescence 27: $415-428$.

Derogatis LR (1993) BSI Bibliography. Minneapolis, MN: National Computer Systems.

Edens JF, Pythress NG, Watkins MM (2001) Further validation of the psychopathic personality inventory among offenders: personality and behavioral correlates. Journal of Personal Disorders 15: 403-415.

Elliot DS, Huizinga D, Menard S (1989) Multiple Problem Youth: Delinquency, Substance Abuse, and Mental Health Problems. New York: Springer-Verlag.

Grisso T, Barnum R (2000) Massachusetts Youth Screening Instrument-Second Version. User's Manual and Technical Reports. Boston, MA: University of Massachusetts Medical School.

Harrell FE (2002) Hmisc and Design libraries. http://biostat.mc.vanderbilt.edu/twiki/bin/view/ Main/RS [15 October 2007].

Hayman-Abello S, Rourke B, Fuerst D (2003) Psychosocial status after pediatric traumatic brain injury: a subtype analysis using the Child Behavior Checklist. Journal of the International Neuropsychological Society 9: 887-898.

Huizinga D, Elliot DS (1986) Reassessing the reliability and validity of self-report delinquency measures. Journal of Quantitative Criminology 2: 293-327.

Hux K, Bong V, Skinner S, Belau D, Sanger D (1998) Parental report of occurrences and consequences of traumatic brain injury among delinquent and non-delinquent. Brain Injury 12: 667-681.

Jolliffe D, Farrington DP, Hawkins JD, Catalano RF, Hill KG, Kosterman R (2003) Predictive, concurrent, prospective, and retrospective validity of self-reported delinquency. Criminal Behaviour and Mental Health 13: 179-197.

Kraus J, Nourjah P (1988) The epidemiology of mild, uncomplicated brain injury. Journal of Trauma 28: 1637-1643.

Krischer MK, Sevecke K, Lehmkuhl G, Rukrop R (2007) Dimensional assessment of personality pathology in female and male delinquents. Journal of Personality Disorders 21: 675-689.

Langlois J, Rutland-Brown W, Thomas K (2006) Traumatic Brain Injury in the United States: Emergency Department Visits, Hospitalizations, and Deaths. Atlanta, GA: Centers for Disease Control and Prevention, National Center for Injury Prevention.

Lilienfeld SO, Andrews BP (1996) Development and preliminary validation of a self-report measure of psychopathic personality traits in noncriminal populations. Journal of Personality Assessment 66: 488-524. 
Maxfield MG, Weiler BL, Widom CS (2000) Comparing self-reports and official records of arrests. Journal of Quantitative Criminology 16: 87-110.

McAllister T (1992) Neuropsychiatric sequelae of head injuries. Psychiatric Clinics of North America 15: 395-412.

Miller J, Jones P (1985) The work of a regional head injury service. Lancet 1: 1141-1144.

Miura H, Fujiki M, Shibata A, Ishikawa K (2005) Influence of history of head trauma and epilepsy on delinquents in a juvenile classification home. Psychiatry and Clinical Neurosciences 59: 661-665.

Mosenthal AC, Livingston DH, Lavery RF, Knudson MM, Lee S, Morabito D, Manley GT, Nathens A, Jorkovich G, Hoyt DB, Coimbra R (2004) The effect of age on functional outcome in mild traumatic brain injury: 6-month report of a prospective multicenter trial. Journal of Trauma 56: 1042-1048.

Rantakallio P, Koiranen M, Mottonen J (1992) Association of prenatal events, epilepsy, and central nervous system trauma with juvenile delinquency. Archives of Disease in Childhood 67: 1459-1461.

Rimel R, Giordani B, Barth J (1981) Disability caused by minor head injury. Neurosurgery 9: 221-228.

Rivara J, Jaffe K, Polissar N, Fay G, Martin K, Shurtleff H, Liao S (1994) Family functioning and children's academic performance and behavior problems in the year following traumatic brain injury. Archives of Physical Medicine and Rehabilitation 75: 369-379.

Address correspondence to: Brian E. Perron, University of Michigan, School of Social Work, 1080 S. University Avenue, Ann Arbor, MI 48109, USA. Tel: +1 734 763-9272. Email: beperron@umich.edu 